\title{
Relationship between blood lactic acid, blood procalcitonin, C-reactive protein and neonatal sepsis and corresponding prognostic significance in sick children
}

\author{
YONGFENG JIA $^{1}$, YING WANG ${ }^{2}$ and XINHUA YU ${ }^{3}$ \\ ${ }^{1}$ Neonatal Department, Shangluo Center Hospital, Shangluo, Shaanxi 721000; \\ ${ }^{2}$ Pediatric Internal Medicine, Weifang People's Hospital, Weifang, Shandong 261041; \\ ${ }^{3}$ Department of Pediatrics, Hubei Institute for Nationalities Affiliated Hospital, Enshi, Hubei 445000, P.R. China
}

Received July 22, 2016; Accepted May 22, 2017

DOI: $10.3892 /$ etm.2017.4713

\begin{abstract}
The study was conducted to evaluate the relationship between the blood lactic acid (BLA) level, serum procalcitonin (PCT), C-reactive protein (CRP) and the severity and prognosis of neonatal sepsis. A retrospective analysis was conducted on 90 children with sepsis admitted into the Intensive Care Unit (ICU) of the Hubei Institute for Nationalities Af liated Hospital hospital. Patients were divided into the non-survival group and the survival group. Severity of the 90 patients was evaluated according to Neonatal Critical Illness Score (NCIS). Observations were made on changes of the levels of BLA, PCT and CRP, correlation between BLA, PCT, CRP and NCIS as well as the association of the levels of these proteins with the prognosis of the patients. The 90 sick children were divided into the survival group (61 cases, 67.7\%) and the non-survival group (29 cases, 32.2\%). They were further stratified into the extremely severe group $(n=20)$, severe group $(n=39)$ and non-severe group $(n=31)$ according to NCIS scoring standard. The BLA and NCIS scores of the non-survival group were significantly greater than those of the survival group. The difference was statistically significant $(\mathrm{P}<0.05)$. We found that there was a significant negative correlation between the BLA values and NCIS scores of the two groups. We also demonstrated significant positive correlation between the BLA value, PCT and CR $(\mathrm{P}<0.05)$. We observed a significant negative correlation between PCT, CRP and NCIS scores $(\mathrm{P}<0.05)$. The PCT level of the non-survival group was significantly higher than that of the survival group $(\mathrm{P}<0.05)$, while the NCIS score was significantly lower than that of the survival group $(\mathrm{P}<0.05)$. The CRP and PCT protein expression results of the sepsis
\end{abstract}

Correspondence to: Dr Xinhua Yu, Department of Pediatrics, Hubei Institute For Nationalities Affiliated Hospital, 2 Wufengshan Road, Enshi, Hubei 445000, P.R. China

E-mail: yuxinhua20160602@163.com

Key words: neonatal sepsis, blood lactic acid, procalcitonin, C-reactive protein, Neonatal Critical Illness Score patients were higher than those of the control group. Therefore, there is a significant correlation between BLA, CRP, PCT and NCIS. The lower the NCIS score is, the more significant the increase of BLA, PCT and CRP. Thus, the combined detection of levels of BLA, PCT and CRP may predict the severity of neonatal sepsis patients and their prognosis.

\section{Introduction}

Currently, sepsis is the main cause of mortality in the Intensive Care Unit (ICU). Clinically, it is important to evaluate the severity and prognosis of neonatal sepsis children at the early stage (1). Generally, neonatal sepsis has two patterns of manifestation: The first is termed 'early-onset sepsis' and refers to when a child is infected within 7 days after birth, and the other is 'late-onset sepsis' and refers to when a child is infected over 7 days after birth. There have been a few studies conducted on the influence of early stage blood lactic acid (BLA) detection on neonatal sepsis (2). In the last 10 years, procalcitonin (PCT) has been proposed as a potential marker due to the fact that it is a type of protein related to sepsis. In addition, the rising levels of PCT bear a close relationship with the morbidity of infectious diseases. C-reactive protein (CRP) is an inflammatory marker and directly participates in the inflammatory process (3-5).

Our study evaluated the clinical significance of early stage dynamic monitoring of the BLA levels, PCT and CRP on the judgment of the severity and prognosis of serious neonatal sepsis by retrospective analysis of the clinical data of the neonatal sepsis children admitted to the ICU with reference to Pediatric Risk of Mortality (PRISM) and Neonatal Critical Illness Score (NCIS). A comparison of liver morphology between normal and pyemia patients was also performed.

\section{Patients and methods}

Patient selection. From January 2014 to December 2015, 90 neonatal patients with sepsis were admitted to the ICU of Hubei Institute for Nationalities Affiliated Hospital (Hubei, China). Inclusion criteria included patients that were diagnosed with sepsis according to the standard guidelines of the neonatal septic shock diagnosis and treatment scheme as 
recommended by the First-aid Group of Chinese Pediatric and Chinese Medical Association and patients that had a predictive survival time of over $24 \mathrm{~h}$. Exclusion criteria were: children born with an inborn error of metabolism, patients that abandoned treatment, and those with incomplete clinical data were not included into this study. The patient cohort comprised 60 men and 30 women with an age range of 3-28 days.

Methods. We recorded the $24 \mathrm{~h}$ BLA values of the 90 patients at the time of admission into the PICU. BLA was measured with Vitros 250 full automation dipsticks analyzer manufactured by Johnson \& Johnson (New Brunswick, NJ, USA). The venous blood was frozen for inspection. NCIS scale was used for the 90 patients in a retrospective manner. VIDAS BRAHMS PCT kit by bioMérieux (Marcy l'Etoile, France) was used for PCT. Enzyme-linked fluorescence assay (ELFA) technology was applied with the reference value of $<0.5 \mathrm{ng} / \mathrm{ml}$ for newborns between 3 and 28 days. Boditech Med Inc. (Chuncheon, Korea) high-sensitivity C-Reactive Protein detection kit was used for high-sensitivity CRP. Immunofluorescence was applied with newborn reference value of $\leq 2 \mathrm{mg} / \mathrm{l}$. Moreover, blood routine examination of the patients was carried out. Special Protein Analyzer manufactured by SEAC (Florence, Italy) was used for CRP inspection. Immune scatter turbidimetry method was used with a normal value of $0-0.8 \mathrm{mg} / \mathrm{dl}$. The study started from the time when the patient was admitted into the Emergency ICU and ended at the time when the patient was discharged from the hospital or succumbed to the disease. Comparison was made on the difference of the BLA level, CRP and PRISM scores of the two groups.

Hematoxylin and eosin (H\&E) staining. For the histopathological observations at the light microscopic level, fresh samples were immersed and fixed in formalin. Following overnight fixation, the specimens were dehydrated in ascending grades of alcohol, cleared in benzene, and embedded in paraffin wax. Blocks were created and 5- to 7- $\mu$ m sections were double-stained with $\mathrm{H} \& \mathrm{E}$ and observed under a light microscope (BX-42; Olympus, Tokyo, Japan).

Western blot analysis. Western blotting was carried our in a routine manner. The gel, Whatman filter sheets (cut approximately equal to the size of the gel) and foam pads were soaked in transfer buffer for equilibration prior to elecro-transfer. The PVDF membrane was also soaked in methanol briefly for 10 sec for activation and then kept in transfer buffer for equilibration. The sandwich of gel and membrane was prepared between the filter sheets covered by foam pads on each side. The gel was kept at the cathode side and membrane at the anode side of the unit. The sandwich was properly tightened with the use of provided screws and placed in the transfer chamber, filled with chilled transfer buffer. Transfer was carried out at $125 \mathrm{~mA}$ for $2 \mathrm{~h}$.

After transfer, the membrane was washed with PBS for 10 min and then placed in blocking buffer $(0.1 \%$ BSA and $0.01 \%$ Tween-20 in PBS) for $1 \mathrm{~h}$ by constant agitation. The membrane was incubated with thefollowing polyclonal antibodies separately: Rabbit polyclonal BLA antibody (dilution, 1:500; cat. no. ab70525); rabbit polyclonal PCT antibody (dilution, 1:500; cat. no. ab121876); rabbit polyclonal CRP antibody (dilution, 1:500; cat. no. ab31156) and rabbit polyclonal $\beta$-actin antibody (dilution, 1:1,000; cat. no. ab8227) were all purchased from Abcam (Cambridge, MA, USA). Dilutions were made with the blocking buffer and the incubation time was $12 \mathrm{~h}$ at $4^{\circ} \mathrm{C}$. The membrane was sequentially washed with PBS, $0.05 \%$ Tween-20 in PBS, and PBS each for 5 min. The membrane was incubated with secondary goat anti-rabbit (eRP) fgd antibody (dilution, 1:2,000; cat. no. ab6721) for $3 \mathrm{~h}$ at $4^{\circ} \mathrm{C}$. Subsequently, the membrane was washed again as described above. The blot was developed in the dark, by adding diaminobenzidine (DAB: $10 \mathrm{mg} / 15 \mathrm{ml}$ PBS containing $15 \mu \mathrm{l}$ of $\mathrm{H}_{2} \mathrm{O}_{2}$ ). The reaction was terminated by rinsing the membrane with double-distilled water and the membrane was air dried.

Statistical analysis. Data were presented using SPSS statistics software version 20 (Chicago, IL, USA). Data are presented as \pm standard deviation. Pearson's correlation analysis was applied. $\mathrm{P}<0.05$ was considered to indicate a statistically significant difference.

\section{Results}

Comparison of liver morphology between normal and pyemia patients. Surgical tissue excision specimen was obtained, fixed, embedded in paraffin and stained with H\&E. The liver morphology of normal tissues appeared normal with the liver blood vessels clearly visible (Fig. 1A). The liver tissue of pyemia patients demonstrated serious liver tissue damage with obscure tissue boundaries (Fig. 1B).

Comparison of the BLA, PCT, CRP and NCIS between the newborn non-survival group and survival group. The 90 patients were divided into the survival group (61 patients, $67.7 \%$ ) and non-survival group (29 patients, 32.2\%). The BLA, PCT and CRP levels of the non-survival group were remarkably higher those of the survival group $(\mathrm{P}<0.05)$. The NCIS of the non-survival group was remarkably lower than that of the survival group $(\mathrm{P}<0.05)$. The difference between the white blood cell (WBC) and neutrophils (NEU) of both groups had no statistical significance (Table I).

Comparison of newborn BLA, PCT, CRP and case fatality rate of different severities. The patients were subdivided into the extreme severe $(n=20)$, severe $(n=39)$ and non-severe $(n=31)$ groups. In terms of the BLA levels, the severe group had lower values than the extremely severe group, and the non-severe group had lower values than both the severe and extremely severe groups $(\mathrm{P}<0.05)$. For PCT levels, the severe group had lower values than the extremely severe group, and the non-severe group had lower values than the severe and extremely severe groups $(\mathrm{P}<0.05)$. For CRP levels, the severe group had lower values than the extremely severe group, and the non-severe group had lower values than both the severe and extremely severe groups $(\mathrm{P}<0.05)$; the difference between the severe and extreme severe groups was statistically significant $(\mathrm{P}<0.05)$. For the NCIS score, the extremely severe group had lower values than the severe and the non-severe groups $(\mathrm{P}<0.05)$. The fatality rate of the extremely severe group was $55 \%$, which was higher than that of both the severe and non-severe groups. The difference was statistically significant 



Figure 1. Liver tissue H\&E staining pathological sections, showing liver paraffin sections of a (A) normal and (B) sepsis patient. H\&E, hematoxylin and eosin.

Table I. Comparison of newborn BLA, PCT, CRP, NCIS, WBC and NEU values.

\begin{tabular}{lccccccc}
\hline Groups & No. of cases & BLA $(\mathrm{mmol} / \mathrm{l})$ & PCT $(\mathrm{ng} / \mathrm{ml})$ & NCIS score & CRP $(\mathrm{mg} / \mathrm{l})$ & WBC $\left(\mathrm{x} 10^{9} / \mathrm{l}\right)$ & NEU $\left(\mathrm{x} 10^{9} / \mathrm{l}\right)$ \\
\hline Survival & 61 & $1.62 \pm 0.84$ & $4.25 \pm 2.40$ & $90.23 \pm 11.12$ & $68.42 \pm 13.22$ & $14.22 \pm 6.2$ & $9.82 \pm 4.22$ \\
Death & 29 & $2.98 \pm 1.57$ & $14.66 \pm 3.72$ & $43.32 \pm 8.50$ & $80.65 \pm 17.42$ & $14.90 \pm 6.11$ & $9.59 \pm 4.48$ \\
t-test & & & 0.95 & 0.94 & 0.95 & 0.65 & 0.72 \\
P-value & & $<0.05$ & $<0.05$ & $<0.05$ & $<0.05$ & $>0.05$ & $>0.05$ \\
\hline
\end{tabular}

BLA, blood lactic acid; PCT, procalcitonin; NCIS, Neonatal Critical Illness Score; CRP, C-reactive protein; NEU, neutrophils.

Table II. Comparison of newborn BLA, PCT, CRP values, NCIS and fatality.

\begin{tabular}{lcccccccc}
\hline Groups & $\begin{array}{c}\text { No. of } \\
\text { cases }\end{array}$ & $\begin{array}{c}\text { BLA } \\
(\mathrm{mmol} / \mathrm{l})\end{array}$ & $\begin{array}{c}\text { PCT } \\
(\mathrm{ng} / \mathrm{ml})\end{array}$ & $\begin{array}{c}\text { CRP } \\
(\mathrm{mg} / \mathrm{l})\end{array}$ & $\begin{array}{c}\text { WBC } \\
\left(\mathrm{x} 10^{9} / \mathrm{l}\right)\end{array}$ & $\begin{array}{c}\text { NEU } \\
\left(\mathrm{x} 10^{9} / \mathrm{l}\right)\end{array}$ & NCIS & $\begin{array}{c}\text { Fatality, } \\
\mathrm{n}(\%)\end{array}$ \\
\hline Extremely severe & 20 & $3.02 \pm 0.55$ & $13.2 \pm 4.37$ & $75.13 \pm 50.87$ & $12.23 \pm 5.97$ & $8.88 \pm 2.52$ & $46.2 \pm 14.87$ & $11(55)$ \\
Severe & 39 & $2.33 \pm 0.98$ & $6.2 \pm 3.24$ & $68.21 \pm 40.23$ & $13.65 \pm 7.21$ & $9.66 \pm 2.67$ & $81.2 \pm 6.33$ & $5(12.8)$ \\
Non-severe & 31 & $1.78 \pm 0.67$ & $2.73 \pm 1.22$ & $16.2 \pm 8.45$ & $14.57 \pm 6.97$ & $9.58 \pm 2.12$ & $94.5 \pm 3.63$ & $1(3.2)$ \\
\hline
\end{tabular}

BLA, blood lactic acid; PCT, procalcitonin; CRP, C-reactive protein; NCIS, Neonatal Critical Illness Score; NEU, neutrophils.

$(\mathrm{P}<0.05)$. The comparison of the WBC and NEU among the three groups had no statistical significance $(\mathrm{P}>0.05)$ (Table II).

Correlation of BLA, PCT, CRP and NCIS scores. There was an obvious positive correlation between BLA, PCT and CRP levels (Fig. 2A) $\left(\mathrm{R}^{2}=0.67, \mathrm{P}<0.05\right)$, while there was an obvious negative correlation between BLA, PCT, CRP and NCIS levels $(\mathrm{P}<0.05)$. There was no correlation between PCT, WBC, and NEU levels ( $P>0.05)$. Therefore, the monitoring of BLA, PCT and CRP levels at early stage are the main factors that can be used to judge the severity of the neonatal sepsis and a favorable method to determine prognosis. The expression results of CRP and PCT protein of the sepsis patients were higher than the normal group (Fig. 2B). Therefore, the BLA, PCT and CRP levels can be used to judge the severity of the serious pediatric sepsis.

\section{Discussion}

Sepsis refers to the systemic inflammatory response syndrome that is caused by infection and is accompanied by circulatory



Figure 2. (A) Correlation between PCT and CRP. (B) Expression results of PCT and CRP protein of sepsis patient. $\mathrm{PCT}$, procalcitonin; $\mathrm{CRP}, \mathrm{C}$-reactive protein. 
dysfunction and organ damage in severe cases (6). The present study investigated the liver protein expression levels of normal people and uremia patients as well as the high expression of the PCT and CRP protein of uremic children. Lactic acid is the metabolite of the anaerobic oxidation of sugar (glycolysis) (7-10) and is generated by the skeletal, muscle, brain and red blood cells. It is excreted by the kidney after liver metabolism. Lactic acid is the normal product of in vivo anaerobic glycolysis. The normal BLA value in humans is 1.0-2.0 mmol/1 (11-13). BLA detection may be used to determine a patient's tissue oxygen delivery, metabolic status as well as insufficient perfusion volume. According to a study that analyzed 52 different shock cases, if the BLA level is lower than $1.4 \mathrm{mmol} / \mathrm{l}$, the fatality is 0 ; if the BLA is lower than $4.4 \mathrm{mmol} / 1$, the fatality is $22 \%$; if the BLA is lower than $8.7 \mathrm{mmol} / 1$, the fatality is $78 \%$; and if the BLA is higher than $13 \mathrm{mmol} / \mathrm{l}$, the fatality is $100 \%$. Therefore, it can be concluded that the amount of lactic acid generation is correlated to oxygen debt, hypoperfusion level and the severity of the shock (14-16).

Currently, WBC count is one of the conventional indexes for clinical neonatal sepsis. However, an increase in WBC counts is not specific. For example, a standard WBC elevation in newborns, acute hemorrhage, virus infection and vomit may all cause an increase in WBC counts (17). In addition, some medication such as analgesic-antipyretic medicine may reduce the WBC count. The present findings showed that the WBC count has no significant correlation with neonatal sepsis. Therefore, WBC is not suitable to be an indicator for auxiliary diagnosis of neonatal sepsis. It can, however, be used as an indicator for auxiliary diagnosis of bacterial infection. CRP is an acute reaction phase protein. CRP levels increase in case of infection (18) as well as in a small number of neonatal noninfectious diseases such as serious gestation infection risk (19-22), use of cortisol parahormone (23), meconium inhalation syndrome and excessive high body mass of premature infant. PCT is also an acute phase reactant with respective features of phase response protein, hormones and cytokines. Neonatal infection, intracranial hemorrhage, fetus antenatal asphyxia, RDS, hypoxemia, hemodynamic disorder, pneumothorax, resuscitation of newborn and gestational diabetes mellitus may all result in the increase of PCT levels (24-27). Thus, the laboratory detection indicators of BLA, PCT and CRP in the auxiliary diagnosis of neonatal sepsis is superior to WBC count.

The results of the present study indicate that the levels of BLA, PCT and CRP can be used to judge the severity of neonatal sepsis and can predict the prognosis of the neonatal sepsis patients to some extent. The higher the PCT level is, the more serious the infection level is, leading to a poorer prognosis. CRP is an acute phase protein and a non-specific anti-inflammatory cytokine, the level of which is low in normal newborn infants. In case of bacterial infection and stress reaction to the body, the CRP levels will rise accordingly. For neonatal sepsis, the change of CRP will be far earlier than the change of temperature or peripheral white blood cell count. Once the infection status is controlled, the CRP levels will decrease quickly (28). According to current research, the CRP level of severe neonatal sepsis patients is increased more significantly than the non-severe patients; however, there is no difference between the non-survival group and the survival group. Therefore, the CRP levels may reflect the severity of the neonatal sepsis patients to some extent and offer prediction on the prognosis of the neonatal sepsis patients. However CRP lacks specificity and sensitivity when compared to PCT (29). Another study has confirmed that the accuracy of WBC in diagnosis of bacterial infection is relatively low and cannot reflect the prognosis (18). The reason is that the range of normal $\mathrm{WBC}$ values is relatively wide with relatively great difference among individuals and may easily be influenced by multiple factors such as other diseases, mental status and sports. The WBC level may also be lowered in case of serious infection.

Currently, NCIS is the most widely-used, objective, easy and effective neonatal critical illness scoring method that is domestically accepted $(30,31)$. However, for neonatal sepsis patients, there is still some limitation to evaluating the severity and predicting the prognosis of patients only with NICS. This study combines the detection of PCT and CRP with NCIS, which can more accurately judge the severity of the neonatal sepsis patients and predict the fatality risks. The neonatal sepsis patients were divided into survival group and non-survival group according to the clinical results. The result indicated that the PCT of the non-survival group was significantly greater than the survival group and the NCIS was significantly lower than the survival group. It was found that PCT had an obvious correlation with NCIS and CRP, while WBC had no correlation with NEU. Therefore, the combination serum PCT, CRP and NCIS may be used to judge the severity of neonatal sepsis and serve as an important indicator of the prognosis.

\section{References}

1. Singhal D, Kumar N, Puliyel JM, Singh SK and Srinivas V: Prediction of mortality by application of PRISM score in intensive care unit. Indian Pediatr 38: 714-719, 2001.

2. Thaver D and Zaidi AK: Burden of neonatal infections in developing countries: areview of evidence from community-based studies. Pediatr Infect Dis J 28 (Suppl): S3-S9, 2009.

3. Ma QQ1, Yang XJ, Yang NQ, Liu L, Li XD, Zhu K, Fu Q and Wei P: Study on the levels of uric acid and high-sensitivity C-reactive protein in ACS patients and their relationships with the extent of the coronary artery lesion. Eur Rev Med Pharmacol Sci 20: 4294-4298, 2016.

4. Qiao W, Yang T and Yang Y: Diagnostic significance of PCT for neonatal sepsis. Int J Lab Med 36: 913-917, 2015 (In Chinese).

5. Ai YH: Treatment of sepsis. Chin J Infect Control 7: 293-297, 2008 (In Chinese).

6. Yuki K and Murakami N: Sepsis pathophysiology and anesthetic consideration. Cardiovase Hematol Disord Drug Targets 15: 57-69, 2015

7. Luzzani A, Polati E, Dorizzi R, Rungatscher A, Pavan R and Merlini A: Comparison of procalcitonin and C-reactive protein as markers of sepsis. Crit Care Med 31: 1737-1741, 2003.

8. Morven SE: Postnatal bacterial in infections. In: Neonatal-Perinatal medicine. Martin RJ, Fanaroff AA and Walsh MC (eds). 9th edition. Mosby Elsevier, St. Louis, MO, pp793-806, 2011.

9. Mussap M, Degrandi R, Cataldi L, Fanos V and Plebani M: Biochemical markers for the early assessment of neonatal sepsis: the role of procalcitonin. J Chemother 19 (Suppl 2): 35-38, 2007.

10. Zamora J, Abraira V, Muriel A, Khan K and Coomarasamy A: Meta-DiSc: a software for meta-analysis of test accuracy data. BMC Med Res Methodol 6: 31, 2006.

11. Yu ZB, Han SP and Guo XR: Meta-analysis of the value of nuchal translucency as a screening test for fetal abnormalities In the first trimester ultrasound examination. Chin J Evid Based Pediatr 3: 407-418, 2008 (In Chinese). 
12. López Sastre JB, Solís DP, Serradilla VR, Colomer BF and Cotallo GD; Grupo de Hospitales Castrillo: Evaluation of procalcitonin for diagnosis of neonatal sepsis of vertical transmission. BMC Pediatr 7: 9, 2007.

13. Ucar B, Yildiz B, Aksit MA, Yarar C, Colak O, Akbay Y and Colak E: Serum amyloid A, procalcitonin, tumor necrosis factor-alpha, and interleukin-1beta levels in neonatal late-onset sepsis. Mediators Inflamm 2008: 737141, 2008.

14. Naher BS, Mannan MA, Noor K and Shahiddullah M: Role of serum procalcitonin and $\mathrm{C}$-reactive protein in the diagnosis of neonatal sepsis. Bangladesh Med Res Counc Bull 37: 40-46, 2011.

15. Gotoff SP: Infection of the neonatal infant. In: Nelson Textbook of Pediatrics. Behrman RE, Kliegman RM and Jenson HB (eds) 16th edition. W.B. Saunders Company, Philadelphia, PA, pp538-549, 2000.

16. Narasimha A and Harendra Kumar ML: Significance of hematological scoring system (HSS) in early diagnosis of neonatal sepsis. Indian J Hematol Blood Transfus 27: 14-17, 2011.

17. Banerjee A, Kelly KB, Zhou HY, Dixon SD, Papana Dagiasis A, Quinn LM and Claridge JA: Diagnosis of infection after splenectomy for trauma should be based on lack of platelets rather than white blood cell count. Surg Infect (Larchmt) 15: 221-226, 2014.

18. Shah BA and Padbury JF: Neonatal sepsis: an old problem with new insights. Virulence 5: 170-178, 2014

19. Reier-Nilsen T, Farstad T, Nakstad B, Lauvrak V and Steinbakk M: Comparison of broad range 16S rDNA PCR and conventional blood culture for diagnosis of sepsis in the newborn: a case control study. BMC Pediatr 9: 5-12, 2009.

20. Abdollahi A, Shoar S, Nayyeri F and Shariat M: Diagnostic value of simultaneous measurement of procalcitonin, interleukin- 6 and hs-CRP in prediction of early-onset neonatal sepsis. Mediterr J Hematol Infect Dis 4: e2012028, 2012.

21. Shahian M, Pishva N and Kalani M: Bacterial etiology and antibiotic sensitivity patterns of early-late onset neonatal sepsis among newborns in Shiraz, Iran 2004-2007. IJMS 3: $293-298,2010$
22. Benitz WE: Adjunct laboratory tests in the diagnosis of early-onset neonatal sepsis. Clin Perinatol 37: 421-438, 2010

23. López Sastre JB, Pérez Solís D, Roqués Serradilla V, Fernández Colomer B, Coto Cotallo GD, Krauel Vidal X, Narbona López E, García del Río M, Sánchez Luna M, Belaustegui Cueto A, et al; Grupo de Hospitales Castrillo: Procalcitonin is not sufficiently reliable to be the sole marker of neonatal sepsis of nosocomial origin. BMC Pediatr 6: 16-19, 2006.

24. Døllner H, Vatten L and Austgulen R: Early diagnostic markers for neonatal sepsis: comparing C-reactive protein, interleukin-6, soluble tumour necrosis factor receptors and soluble adhesion molecules. J Clin Epidemiol 54: 1251-1257, 2001.

25. Vergnano S, Menson E, Kennea N, Embleton N, Russell AB, Watts T, Robinson MJ, Collinson A and Heath PT: Neonata infections in England: the NeonIN surveillance network. Arch Dis Child Fetal Neonatal Ed 96: F9-F14, 2011.

26. Kujari AM, Waris M, Lehtonen L and Ruuskanen O: Respiratory viral infections are not uncommon in neonatal intensive care units. Acta Paediatr 103: e225-e228, 2014.

27. Marchant EA, Boyce GK, Sadarangani M and Lavoie PM: Neonatal sepsis due to coagulase-negative staphylococci. Clin Dev Immunol: doi 2013: 586076, 2013.

28. Vazzalwar R, Pina-Rodrigues E, Puppala BL, Angst DB and Schweig L: Procalcitonin as a screening test for late-onset sepsis in preterm very low birth weight infants. J Perinatol 25: 397-402, 2005.

29. Arnon S and Litmanovitz I: Diagnostic tests in neonatal sepsis. Curr Opin Infect Dis 21: 223-227, 2008.

30. Altunhan H, Annagür A, Örs R and Mehmetoğlu I: Procalcitonin measurement at 24 hours of age may be helpful in the prompt diagnosis of early-onset neonatal sepsis. Int J Infect Dis 15: e854-e858, 2011.

31. Yu Z, Liu J, Sun Q, Qiu Y, Han S and Guo X: The accuracy of the procalcitonin test for the diagnosis of neonatal sepsis: a meta-analysis. Scand J Infect Dis 42: 723-733, 2010. 